\title{
Soliton-pair Propagation under Thermal Bath Effect
}

\author{
N. Boutabba ${ }^{1,3}$, H. Eleuch ${ }^{1,2,3 *}$ \\ ${ }^{1}$ Department of Physics and Astronomy, Texas A\&M University College Station, Texas 77843, USA \\ ${ }^{2}$ Max Planck Institute for Physics of Complex Systems, 01187 Dresden, Germany \\ ${ }^{3}$ Université of Carthage, Tunis, Tunisia
}

\begin{abstract}
We consider two atomic transitions excited by two variable laser fields in a threelevel system. We study the soliton-pair propagation out of resonance and under thermal bath effect. We present general analytical implicit expression of the soliton-pair shape. Furthermore, we show that when the coupling to the environment exceeds a critical value, the soliton-pair propagation through three-level atomic system will be prohibited.
\end{abstract}

Keywords and phrases: quantum optics, non linear optics

Mathematics Subject Classification: 81V80, 78M99

\section{Introduction}

Recently the interaction of atoms or similar systems with electromagnetic fields has raised a lot of interest $[6,7,11,13-18,22,25,26,32]$. It leads to interesting quantum features such as, entanglement [33], antibunching [19], squeezing [30], bistability [3] and optical soliton propagation [20,21]. Solitons arise as the solutions of a widespread class of weakly nonlinear dispersive partial differential equations describing physical systems. They are an essential nonlinear kind of wave-like excitation, caused by a cancellation of nonlinear and dispersive effects in the medium and they have particle-like properties. Solitons have important applications in many branches of physics, from high energy and condensed matter physics to astrophysics and cosmology as well as in biology and telecommunication [4,9,10,27,29,34]. Early work, have been investigated $[8,20,31]$ to computed the properties of solitons.

In the $\Lambda$ configuration, a pair of optical pulses propagate without absorption. This medium can be made experimentally [5] if two lasers are applied to a three-level system, the atoms will be driven to a population trapped state, and a medium that is opaque to a probe laser can, by applying both lasers simultaneously, be made transparent $[2,23]$. In previous work $[8,20,21]$, we derived analytical solutions of solitons and pair of solitons in dissipative media.

In this paper we investigate the Soliton-pair propagation in the three-level dissipative media out of resonance, under thermal bath effect.

\footnotetext{
${ }^{*}$ Corresponding author. E-mail: heleuch@fulbrightmail.org
} 


\section{Model}

Let us consider a three-level system in lambda configuration ( three-level atom ) interacting with two non resonant electromagnetic fields. The medium is excited by two laser fields one applied on the stokes transition and the second on the pump transition.

This system is described with three energy levels $|0\rangle,|1\rangle$ and $|2\rangle$. The transitions $|0\rangle \leftrightarrow|1\rangle$ and $|0\rangle \leftrightarrow|2\rangle$ are possible whereas the levels $|1\rangle$ and $|2\rangle$ are supposed to be decoupled (transition $|1\rangle \leftrightarrow|2\rangle$ negligible). The reason of the choice of this model is the fact that a free atom has at least two states at same parity between which an electrical dipole transition is not allowed. The restriction to two lower energy level is valid if the frequency of the interacting waves are distant enough to all other frequencies. In this model we take into account the rates $\gamma_{1,2}$ of radiative decay from the higher level $|0\rangle$ to the levels $|1\rangle$ and $|2\rangle$ and neglecting the other dissipation effects.

This three-level system is irradiated by a light beam propagating along an arbitrary direction $\mathrm{x}$, with polarization adequate to couple the two optical transitions, and containing two monochromatic fields. This light beam is classically described as follows:

$$
E^{\prime}(x, t)=E_{1}(x, t)+E_{2}(x, t)=\bar{E}_{1}(x, t) \exp \left(-i \omega_{1} t\right)+\bar{E}_{2}(x, t) \exp \left(-i \omega_{2} t\right) .
$$

$\bar{E}_{1}$ and $\bar{E}_{2}$ are the amplitudes of the two waves. $\bar{E}_{1}$ and $\bar{E}_{2}$ are assumed to be slowly varying functions in the sense [12], [28], [1]: $\frac{1}{\omega_{2}}\left|\frac{\partial \bar{E}_{2}}{\partial t}\right|<<\left|\bar{E}_{2}\right|$ and $\frac{1}{\omega_{1}}\left|\frac{\partial \bar{E}_{1}}{\partial t}\right|<<\left|\bar{E}_{1}\right|$.

The Hamiltonian describing the interaction of the three level atom with the field has the expression:

$$
H=\sum_{i=0}^{2} \varepsilon_{i} a_{i}^{+} a_{i}+g_{1}\left(a_{0}^{+} a_{1} E_{1}+a_{1}^{+} a_{0} E_{1}^{*}\right)+g_{2}\left(a_{0}^{+} a_{2} E_{2}+a_{2}^{+} a_{0} E_{2}^{*}\right) .
$$

The first term of the Hamiltonian corresponds to the proper energies of the atom, the second and third terms of the Hamiltonian describe the interaction between the two fields and the atom: $a_{i}, a_{i}^{+}$ are respectively the annihilation and creation fermions operators of the atomic level $\mathrm{i}$ and $\varepsilon_{i}$ represent the energy of the levels i. $a_{i}, a_{i}^{+}$verify the anticommutation relation $\left[a_{i}, a_{j}^{+}\right]_{+}=\delta_{i j}$. The two dipole transition matrix elements which are assumed to be real are denoted by $g_{1}$ and $g_{2}$.

To study the evolution of interaction between the atom and fields we use the density matrix formalism. The density matrix equation of motion is

$$
\frac{d}{d t} \rho=\frac{1}{i \hbar}[H, \rho]+\frac{d}{d t} \rho_{i r r},
$$

where $\frac{d}{d t} \rho_{i r r}$ describes the dissipation in the total system and the coupling to the thermal reservoir.

$$
\begin{aligned}
\frac{d}{d t} \rho_{\text {irr }}= & \frac{\gamma_{1}}{2}\left(1+n_{t h}\right)\left(\left[a_{1}^{+} a_{0}, a_{0}^{+} a_{1} \rho\right]+\left[a_{1}^{+} a_{0} \rho, a_{0}^{+} a_{1}\right]\right) \\
& +\frac{\gamma_{1}}{2} n_{t h}\left(\left[a_{0}^{+} a_{1}, \rho a_{1}^{+} a_{0}\right]+\left[a_{0}^{+} a_{1} \rho, a_{1}^{+} a_{0}\right]\right) \\
& \frac{\gamma_{2}}{2}\left(1+n_{t h}\right)\left(\left[a_{2}^{+} a_{0}, a_{0}^{+} a_{2} \rho\right]+\left[a_{2}^{+} a_{0} \rho, a_{0}^{+} a_{2}\right]\right) \\
& +\frac{\gamma_{2}}{2} n_{t h}\left(\left[a_{0}^{+} a_{2}, \rho a_{2}^{+} a_{0}\right]+\left[a_{0}^{+} a_{2} \rho, a_{2}^{+} a_{0}\right]\right) .
\end{aligned}
$$

So, we obtain the motion equation of the density matrix: where the elements $\rho_{i j}$ are defined as $\rho=$ $\sum_{i, j=0}^{2}|i\rangle \rho_{i j}\langle j|, \omega_{10}$ and $\omega_{20}$ represent the two atomic transition frequencies $\omega_{10}=\frac{\varepsilon_{0}-\varepsilon_{1}}{\hbar}$ and $\omega_{20}=\frac{\varepsilon_{0}-\varepsilon_{2}}{\hbar}$ and $d_{i}=\frac{g_{i}}{\hbar}$ are the coupling constants. The diagonal elements of the density matrix $\rho$ describe the 
level populations and determine the internal energy of the atom. The off-diagonal elements describe the atomic coherences. The $\rho_{10}$ and $\rho_{20}$ terms oscillate at the respective driving field frequency and the $\rho_{21}$ oscillate with frequency differences of the two light fields. So, we can define the slowly varying amplitudes of the off-diagonal density matrix elements $\rho_{10}^{-}, \overline{\rho_{20}}$ and $\rho_{21}^{-}$through the relations:

$$
\begin{aligned}
& \rho_{j 0}=\rho_{j 0}^{-} \exp \left(i \omega_{j 0} t\right) \quad \text { for } j=1,2, \\
& \rho_{21}=\rho_{21}^{-} \exp \left(i\left(\omega_{20}-\omega_{10}\right) t\right) .
\end{aligned}
$$

We decompose the off-diagonal elements into an imaginary part and a real part :

$$
\begin{aligned}
& \rho_{j 0}^{-}=\chi_{j 0}+i \psi_{j 0}, \\
& \rho_{21}^{-}=\chi_{21}+i \psi_{21} .
\end{aligned}
$$

The Hermitian propriety of the density matrix ensures that the diagonal elements $\rho_{11}, \rho_{22}$ and $\rho_{00}$ must be real. $\delta_{1}$ and $\delta_{1}$ are the detunings between the laser frequencies and the atomic transitions frequencies: $\delta_{1}=\omega_{10}-\omega_{1}$ and $\delta_{2}=\omega_{20}-\omega_{2}$. The signal field $\mathrm{E}_{2}$ and $\mathrm{E}_{1}$ are described by the Maxwell equations for a slowly varying approximation (SVA) $[2,12]$ :

$$
\frac{\partial \bar{E}_{j}}{\partial t}+c \frac{\partial \bar{E}_{j}}{\partial x}=i g_{j}^{\prime} \rho_{j 0}^{-}
$$

We assume that the propagation constants of the fields are given by $g_{j}^{\prime}=\frac{2 \pi}{\varepsilon_{0}} N g_{j}\left(\omega_{j}+\delta_{j}\right)$ where $j=1,2$ ,$\varepsilon_{0}$ is the vacuum electric constant, $N$ the atomic dipole density and $c$ is the light velocity. The condition for soliton-pair propagation is expressed as $\bar{E}_{j}(x, t)=\bar{E}_{j}\left(x-v_{g} t\right)$. We consider here two fields $\bar{E}_{1}$ and $\bar{E}_{2}$ and we assume that they are real. The fact that $\bar{E}_{2}$ is real gives us $\chi_{20}=0$. Then we introduce a moving coordinate which propagates with the pulses' velocities $z=x-v_{g} t$ which gives us $\frac{\partial}{\partial t}=-v_{g} \frac{\partial}{\partial z}$ and $\frac{\partial}{\partial x}=\frac{\partial}{\partial z}$ where $v_{g}$ can be identified with the group velocity of the soliton-pair. The two spontaneous emission rates $\gamma_{1}$ and $\gamma_{2}$ are assumed to be approximately equal to $\gamma$. Finally, the complete set of the evolution equations for medium-fields interaction ( Maxwell-Bloch equations) in the case of two photon resonance $\delta_{1}=\delta_{2}$ can be obtained from Maxwell equation and the system of evolution equations for the density matrix:

$$
\begin{aligned}
\frac{d}{d z} \chi_{10} & =\frac{\delta}{v_{g}} \psi_{10}-\alpha_{2} \psi_{21}+\Gamma(1+3 n / 2) \chi_{10}, \\
\frac{d}{d z} \psi_{10} & =\frac{\delta}{v_{g}} \chi_{10}-2 \alpha_{1} \chi_{11}-\alpha_{2} \chi_{21}+\Gamma(1+3 n / 2) \psi_{10}, \\
\frac{d}{d z} \psi_{20} & =\alpha_{2}\left(-1-\chi_{11}\right)-\alpha_{1} \chi_{21}+\Gamma(1+3 n / 2) \psi_{20}, \\
\frac{d}{d z} \chi_{21} & =\alpha_{1} \psi_{20}+\alpha_{2} \psi_{10}+\Gamma n \psi_{21}, \\
\frac{d}{d z} \psi_{21} & =\alpha_{2} \chi_{10}+\Gamma n \psi_{21}, \\
\frac{d}{d z} \chi_{11} & =2 \alpha_{1} \psi_{10}+\Gamma \chi_{11}(1+2 n), \\
\frac{d}{d z} \alpha_{j} & =-\frac{d_{j} g_{j}^{\prime}}{v_{g}\left(c-v_{g}\right)} \psi_{j 0}=-k_{j} \psi_{j 0} \quad j=1,2, \\
0 & =\frac{\delta}{v_{g}} \psi_{20}+\alpha_{1} \psi_{21} .
\end{aligned}
$$


Where $\alpha_{j}$ are variables related to the field amplitudes by the following expressions $\alpha_{j}=\frac{d_{j} \bar{E}_{j}}{v_{g}} . \Gamma=\frac{\gamma}{v_{g}}$ represents a new constant .

\section{Soliton-pair shapes}

Our interest is in studying the evolution of the fields $\alpha_{2}$ and $\alpha_{1}$ and we deal with the case of similar shape soliton-pair so we can write $\alpha_{1}=A \alpha_{2}=A \alpha(A$ is a real constant $>1)$. The fields 1 and 2 have a slowly varying amplitudes, in this case, we can neglect the variation of the curvature and we can assume that the third and the forth order of derivation are negligible. After algebraic manipulations and differentiation of the Maxwell-Bloch equations, we obtain a non-linear differential equation:

$$
\left(-A^{2} E-F\right) \alpha^{4}+M \alpha^{2}=-B(1+\alpha) \frac{d \alpha}{d z} .
$$

$E, F, B$ and $M$ are constants depending of the system parameters

$$
\begin{aligned}
E & =\frac{\delta}{v_{g} K_{1}}, \\
B & =\frac{\delta}{v_{g} K_{2}}(1-\Gamma n), \\
M & =\Gamma(1+3 n / 2)(1-\Gamma n) \frac{\delta}{v_{g} K_{2}}, \\
F & =\frac{\delta}{v_{g} K_{2}} .
\end{aligned}
$$

by integrating the above equation, the soliton $\alpha$ verifies the following implicit equation

$$
\eta e^{-2 \frac{M z}{b}}=e^{\frac{-1}{\alpha_{2}(z)}} \alpha_{2}(z)\left(\frac{1+\beta \alpha_{2}(z)}{1-\beta \alpha_{2}(z)}\right)^{1 / 2 \beta}\left(-1+\beta^{2} \alpha_{2}^{2}(z)\right)^{-1 / 2},
$$

where $\beta=\sqrt{\frac{A^{2} E+F}{M}}=\sqrt{\frac{A^{2}-1}{\Gamma(1+n 3 / 2)(1-n \Gamma)}}$ and $\eta$ is a free constant that can be determined from the initial condition as following

$$
\eta=\alpha_{2}\left(z_{0}\right) e^{2 \frac{M z_{0}}{b}-\frac{1}{\alpha_{2}(0)}}\left(\frac{1+\beta \alpha_{2}\left(z_{0}\right)}{1-\beta \alpha_{2}\left(z_{0}\right)}\right)^{1 / 2 \beta}\left(-1+\beta^{2} \alpha_{2}^{2}\left(z_{0}\right)\right)^{-1 / 2} .
$$

This gives us a condition of existence of the signal field

$$
\frac{-1}{\beta}<\alpha<\frac{1}{\beta} \text { and } \alpha \neq 0 .
$$

In other words if the initial amplitude value of the signal $\alpha$ is out of the range $\left[\frac{-1}{\beta}, \frac{1}{\beta}\right]$ no more solitonpair propagation is possible. Moreover the thermal bath coefficient $n$ should be less than a critical value $n_{c}=1 / \Gamma$, otherwise if the coupling to the environment exceeds the critical value $n_{c}$ then no more solitonpair can be propagate. In Figure 1 we plot the soliton shape $\alpha(z)$ for $n=1.5, \Gamma=0.5, E=1$ and $F=1$ and for the initial condition $\alpha(0)=0.05$. 


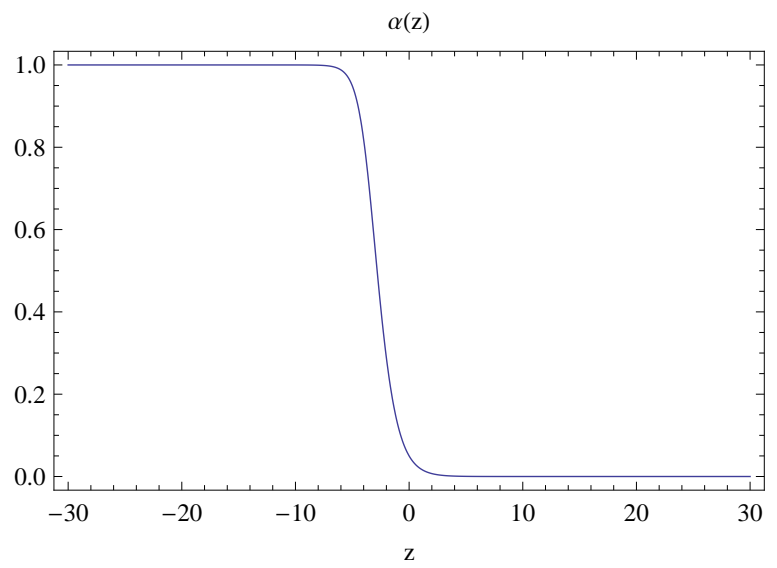

Figure 1. soliton shape for in $n=1.5, \Gamma=0.5, E=1, F=1$ and for the initial condition $\alpha(0)=0.05$.

\section{Conclusion}

In summary, we have investigated a theoretical model describing a pair of solitons propagating through an absorbing three-level atoms, interacting with the environment through the thermal bath effect, out of resonance. We have derived an analytical implicit expression for the shape of the soliton-pair. We have shown that up a critical value of the thermal bath, the soliton-pair is not allowed to propagate through the three-level atomic system.

These results are useful in optical data communication, where the optical fibre can be modelled as an absorbing three level system [5]. The advantage of soliton in supporting data information is the invariance of their shape which minimizes the noise effect, this is usually the origin of the signal defects. Besides, solitons propagate without dispersion. Therefore, we can send the optical information with high bit rate.

\section{References}

[1] L. Allen, J. Eberly. Optical Resonance and Two-Level Atoms. Dover, New York, 1987.

[2] G. Alzetta, A. Gozzini, L. Moi, G. Orriols. Experimental-method for observation of Rf Transitions and Laser beat resonances in oriented Na vapor. Nuova Cimento, 36 (1976), No. 1, 5-20.

[3] A. Baas, J. Karr, H. Eleuch, E. Giacobino. Optical bistability in semiconductor microcavities. Phys. Rev. A, 69 (2004), No. 2, 023809.

[4] A. Bishop, J. Krumhansl, S. Trullinger. Solitons in condensed matter: A paradigm. Physica D, 1 (1980), No. 1, 1-44.

[5] K. Boller, A. Imamogluand, S. Harris. Observation of electromagnetically induced transparency. Phys. Rev. Lett 66 (1991), No. 20, 2593-2596.

[6] N. Boutabba, L. Hassine, A. Rihani, H. Bouchriha. Analytic photocurrent transient response of an Al/6T/ITO photovoltaic cell using Volterra series analysis. Synthetic Metals, 139 (2003), No. 2, 227-231.

[7] N. Boutabba, L. Hassine, N. Loussaief, F. Kouki, H. Bouchriha. Volterra series analysis of the photocurrent in an Al/6T/ITO photovoltaic device. Organic Electronics, 4 (2003), No. 1, 1-8.

[8] N. Boutabba, H. Eleuch, H. Bouchriha. Thermal bath effect on soliton propagation in three level atomic system. Synthetic Metals, 159 (2009), No. 13, 1239-1243.

[9] M. Chernodub, Sh. Hu, A. Niemi. Topological solitons and folded proteins. Phys. Rev. E, 82 (2010), No. $1,011916$.

[10] C. Lechner, S. Husa, C. Aichelburg. SU(2) cosmological solitons. Phys. Rev. D, 62 (2000), No. 4, 044047.

[11] G. Dridi, S. Guerin, V. Hakobyan, H Jauslin, H Eleuch. Ultrafast stimulated Raman parallel adiabatic passage by shaped pulses. Phys. Rev A, 80 (2009), No. 4, 043408.

[12] J. Eberly. Transmission of dressed field in 3-level media. Quantum Semiclass. Opt. 7 (1995), No. 3, $373-384$.

[13] H. Eleuch, N. Rachid. Autocorrelation function of microcavity-emitting field in the non-linear regime. Eur. Phys. J. D., 57 (2010), No. 2, 259-264.

[14] H. Eleuch. Autocorrelation function of microcavity-emitting field in the linear regime. Eur. Phys. J. D., 48 (2008), No. 1, 139-143.

[15] H. Eleuch. Noise spectra of microcavity-emitting field in the linear regime. Eur. Phys. J. D., 49 (2008), No. 3, $391-395$. 
[16] H. Eleuch. Quantum trajectories and autocorrelation function in semiconductor microcavity. Applied Mathematics \& Information Science 3 (2009), No. 3, 185-196.

[17] H. Eleuch, N. Ben Nessib, R. Bennaceur. Quantum Model of emission in weakly non ideal plasma. Eur. Phys. J. D, 29 (2004), No. 3, 391-395.

[18] H. Eleuch, R. Bennaceur. Non linear dissipations and the quantum noise of light in semiconductor microcavities. J. Opt. B: Quantum and Semiclassical Optics, 6 (2004), No. 4, 189-195.

[19] H. Eleuch. Photon statistics of light in semiconductor microcavities. J. Phys. B, 41 (2008), No. $5,055502$.

[20] H. Eleuch, D. Elser, R. Bennaceur. Soliton propagation in an absorbing three level atomic system. Laser Phys. Lett., 1 (2004), No. 8, 391-396.

[21] H. Eleuch, R. Bennaceur. An optical Soliton pair among absorbing three-level atoms. J. Opt. A: Pure Appl.Opt., 5 (2003), No. 5, 528-533.

[22] E. Giacobino, J. Karrr, G. Messin, H. Eleuch. Quantum optical effects in semiconductor microcavities. C. R. Physique, 3 (2002), No. 1, 41-52.

[23] H. Gray, R. Whitley, C. Stroud. Coherent trapping of atomic populations. Optics Letters, 3 (1978), No. 6, 218-220.

[24] Y. Guo, C. Kao, E. Li, K. Chiang. Nonlinear Photonics, Series in Photonics, Springer, New York, 2002.

[25] H. Jabri, H. Eleuch, T. Djerad. Lifetimes of atomic Rydberg states by autocorrelation function. Laser Phys. Lett., 2 (2005), No. 5, 253-257.

[26] P. Jha, H. Eleuch, Y. Rostovtsev. Coherent control of atomic excitation using off-resonant strong few-cycle pulses. Phys. Rev. A, 82 (2010), No. 4, 045805.

[27] G. Korchemsky, I. Krichever. Solitons in high-energy QCD. Nucl. Phys. B, 505 (1997), No.1-2, 387-414.

[28] L. Mandel, E. Wolf. Optical Coherence and Quantum Optics, Cambridge University Press, New York, 1995.

[29] M. Marklund, D. Tskhakaya, P. Shukla. Quantum electrodynamical shocks and solitons in astrophysical plasmas. Europhys. Lett., 72 (2005), No. 6, 950-954.

[30] G. Messin, J. Karr, H. Eleuch, J. Courty, E. Giacobino. Squeezed states and quantum noise of light in semiconductor microcavities. J. Phys.: Condens. Matter, 11 (1999), No. 31, 6069-6078.

[31] Q. Park and H. Shin. Systematic construction of multicomponent optical solitons. Phys. Rev. E, 61 (2000), No. $3,3093$.

[32] Y. Rostovstev, H. Eleuch, A. Svidzinsky, H. Li, V. Sautenkov, M. Scully. Generation of maximal coherence in a 2-level system via breaking of adiabaticity. Phys. Rev. A., 79 (2009), No. 6, 063833.

[33] E. Sete and H. Eleuch. Interaction of a quantum well with squeezed light: Quantum statistical properties. Phys. Rev. A, 82 (2010), No. 4, 043810.

[34] M. Wesner, C. Herden, R. Pankrath, D. Kip, P. Moretti. Temporal development of photorefractive solitons up to telecommunication wavelengths in strontium-barium niobate waveguides. Phys. Rev. E, 64 (2001), No. $3,036613$. 